\title{
ROLE OF WITHANIA SOMNIFERA AND IT'S ACTIVE PRINCIPLE WITHANOLIDE-A IN BALANCE AND MOTOR CO-ORDINATION BY FOOT PRINT TEST
}

\section{A. Mary Antony Praba *1, C.Venkataramaniah ${ }^{2}$, Kavitha Ganesh ${ }^{3}$, Krishna Eswari .}

${ }^{* 1}$ Associate Professor, Department of Anatomy, Tagore Medical College, Chennai, Tamilnadu, India.

${ }^{2}$ Professor, Department of Anatomy, Tagore Dental College, Chennai, Tamilnadu, India.

${ }^{3}$ Reader, Department of Anatomy, Tagore Dental College, Chennai, Tamilnadu, India.

${ }^{4}$ Reader, Department of Anatomy, Tagore Dental College, Chennai, Tamilnadu, India.

\section{ABSTRACT}

Background: The basal ganglia historically been considered as a part of the motor system because of the variety of motor deficits that occur when they are damaged. But now it is considered as "extrapyramidal" motor system, and the disorders of basal ganglia are called extrapyramidal disorders. One type of symptoms that result from basal ganglia disorders is called as Huntington's Chorea. As this disorder involves with symptoms like dyskinesias - abnormal involuntary movements, we felt it is necessary to protect our nervous system from such a disorder if possible in a painless regular fashion by a herb.

Materials and Methods: We used adult male Sprague Dawly rats for this study. Animals were divided into 5 groups and were given either Withania somnifera extract or the active component Withanolide A in different concentrations 10 days prior to lesion surgery and continued 5 days post surgery. The neuroprotective role of the drug employed was analyzed on the $5^{\text {th }}$ day post lesion by using foot print test in a run way.

Result: The gait and balance of the animals were taken as a measure to analyze the protective nature of the striatum and so the activity of the drug employed here. The gait and balance of the LC animals were poor stating the unprotective nature of striatum. But the balance and gait of both drug group animals were comparatively better than the LC animals. That clearly stated the neuroprotective capacity of both the drugs used for this study.

Conclusion: Based on the observations and results we came to a conclusion that both the ethanolic extract and the active component withanolide $A$ have the capacity in protecting the striatum and so can be used as a food supplement on a daily basis to protect our striatum. If needed further research can be conducted to analyze deep into the therapeutic effects of these herbal drugs.

KEY WORDS: Basal ganglia, Extrapyramidal, Huntington's Chorea, Dyskinesias, Withania somnifera, Withanolide A, Gait, Striatum.

Address for Correspondence: Dr. A. Mary Antony Praba. Associate Professor, Department of Anatomy, Tagore Medical College, Chennai, Tamilnadu, India. E-Mail: fio7rio@yahoo.co.in

Access this Article online

Quick Response code

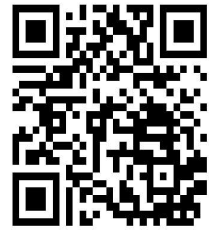

DOI: $10.16965 /$ ijar.2017.424
Journal Information

International Journal of Anatomy and Research

ICV for 2016
90.30 ISSN (E) 2321-4287 | ISSN (P) 2321-8967

https://www.ijmhr.org/ijar.htm

DOI-Prefix: https://dx.doi.org/10.16965/ijar

\section{Article Information}

Received: 17 Aug 2017

Peer Review: 17 Aug 2017

Revised: None
Accepted: 03 Oct 2017

Published (O): 05 Jan 2018

Published (P): 05 Jan 2018

\section{INTRODUCTION}

The basal ganglia (BG) are a highly organized network, where different parts are activated for specific functions like movement control, associative learning, planning, working memory, and emotions [1] by secreting some inhibitory neurotransmittors. In general the basal ganglia 
are associated with a variety of functions, including voluntary motor control, procedural learning related to routine behaviors or "habits" such as bruxism, eye movements, and cognitive, emotional functions [2] by inhibition.

Huntington's disease (HD) is a fatal neurodegenerative disorder that causes defects in behavior, cognition, and uncontrolled rapid, jerky movements. Huntington's disease is associated with basal ganglia degeneration [3]. This degeneration of striatal neurons leads to hyperkinetic movement disorders characterized by increased uncontrollable motor functions due to inability to stop unwanted movement.

Huntington's disease is also known as Huntington's chorea because it is characterized by a continuous, choreiform movements of the body (Greek word for "dance") Helmes and Shulman 2005 [4]. There is no cure for HD, and full-time care is required in the later stages of the disease [5]. The neuronal degeneration and other complications such as pneumonia, heart disease, and physical injury from falls reduce the life expectancy and eventually cause death within 10 to 20 years.

According to World Health Organization (WHO), 80 percent of the world's population presently uses herbal medicine for some aspect of primary health care [6]. Ashwagandha, also called as "Queen of Ayurveda" is a very important plant in Ayurveda, the Indian traditional medicine. This herb was used 4000 years plus in India.

Soman 2012 investigated the effect of Withania somnifera (WS) root extract and Withanolide $A$ (WD) in restoring spatial memory deficit by oxidative stress induced alteration in the hippocampus of epileptic rats and concluded that treatment with WS and WA has ameliorated spatial memory deficits by enhancing antioxidant system.

All the above said conditions paved ways to conduct a study to find the neuroprotective role of Withania somnifera and Withanolide $A$ in experimental Huntington's chorea rats by analyzing the gait difference of animals using a runway.

\section{MATERIALS AND METHODS}

Animals: We used adult male Sprague Dawly rats weighing (200-240 gm) for this study and maximum effort was taken to minimize the unwanted stress to the animals and to reduce the number of animal to be used for this study. Animals were divided into 5 groups with 6 animals in each group. They are control group (CO), lesion control (LC), sham control (SC), withania somnifera ethanolic extract $25 \mathrm{mg} / \mathrm{kg}$ body weight (WS 125) and withanolide A 100mg/ kg body weight (WD 100). The drugs were dissolved in normal saline and the volume was adjusted to $1 \mathrm{ml}$. The administration of drug was started 10 days prior to lesion surgery so as to access the protective role of the drug in striate neurons. Dosages of drugs were given IP. around 10 O' clock every day. (Table-1)

\section{Preparation and administration of the drug:}

Ethanolic Extract of Withania Somnifera (WS)

Preparation: The ethanolic extract was prepared by soxhlation method following Elayaraja et al., 2010(7). He proved that the ethanolic extract expressed more antioxidant activity than other extracts of withania somnifera. The I.P. dosage of this drug was prepared by dissolving the ethanolic extract of the drug in normal saline (Mohan et al., 2008(8)), the volume was adjusted to $1 \mathrm{ml}$ for each animal

Withanolide A: Withanolide A was purchased from Sigma Fluka-USA. The I.P. Oral dosage was avoided to reduce the wastage of drug and to minimize the stress condition of rats. The drug was started 10 days prior to lesion and 5 days post lesion so as to access the protective role and the therapeutic power of it in striatum. Drug administrating time was maintained around 10' clock every day.

FOOT PRINT ANALYSIS FOR GAIT (Carter et al, 1999 [9])

Paw Print is a simple assay to measure gait analysis and balance.

\section{Apparatus (Figure-1)}

The runway apparatus was a elongated box with a walkable $50-\mathrm{cm}$-long, $10-\mathrm{cm}$-wide runway with 10-cm-high walls.

\section{Procedure}

Trial: One run per day per animal for one week was given as trial.

Test: The rats were acclimated in the testing room for $15 \mathrm{~min}$ 
The rats were held in supine position and the limbs were painted with a nontoxic watercolor paint, one color for fore paw and another for hind paw.

The animals were then placed in a clean runway lined with white paper and allowed to explore and run way.

The white sheet with the paw print was taken, the first and the last paw prints were omitted and the remaining prints were analyzed for coordination of gait.

\section{Parameters}

Print length (PL): Distance from the heel to the third toe

Toe spread (TS): Distance from the first to the fourth toe

DOF: Distance to opposite foot.

Gait difference: Distance from left or right front footprint /hind footprint overlap was used to ensure the uniformity of step alternation. When the center of the hind footprint fell on top of the center of the preceding front footprint, a value of zero was recorded. When the footprints did not overlap, the distance between the center of the footprints were recorded.

\section{Scores:}

short print length: Poor balance and gait coordination

low toe spread: Good balance and gait coordination

Less distance to opposite foot: Poor gait coordination

Gait difference: Poor balance and gait coordination

Hippocampal lesion surgery: The animals were maintained in empty stomach $10 \mathrm{~h}$ before the procedure and were anaesthetized using pentathol sodium. The hair in the head region was shaved using diluted savlon as sanitizer. The animals were fixed in the stereotaxic frame with the help of the tooth and nose bar.

A $2 \mathrm{~cm}$ long incision was made along the scalp of the rats and the fascia was cleaned to point out the bregma [10]. Necessary steps were taken to avoid infections at all levels. The striata were marked in the scalp region by moving the manipulator from the bregma $2.2 \mathrm{~mm}$ anteriorly,
$3 \mathrm{~mm}$ bilaterally and a small hole with $1 \mathrm{~mm}$ diameter was made in the marked region.

$0.5 \mu$ l of kainic acid [11] was taken in a Hamilton syringe and was fixed in the frame. With the help of the manipulator the syringe was moved $5 \mathrm{~mm}$ inferiorly from the dura to the striatum. The same chemical was injected in the rate of 1 $\mu$ per 1 minute following Mcginty et al., 1983 [12]. The syringe was withdrawn and the scalp was sutured with proper care.

Post operative care: Proper antibiotic care was given post lesion with $2 \mathrm{mg} / \mathrm{kg} /$ day gentamysin for 3 days

\section{RESULTS AND DISCUSSION}

Foot Print Analysis (figure-1, figure-2): This study was performed to analyse the gait co- ordination and balance of the animals. For this study the fore limb of the animals were painted with fluorescent green colour and the hind limbs were painted with fluorescent pink and made to run in the apparatus. Three parameters were studied to analyse the animal's behaviour.

Toe Spread (figure-3, chart-1): In this parameter the distance from the first to the fourth toe was measured in cm's and analysed. The animals with low toe spread were considered as having good balance and gait coordination according to literatures.

The toe spread of the LC animals were significantly high when compared with the $\mathrm{CO}$ (figure2) animals. The SC group of animals were not showing any significance in their toe spread and was equal with the $\mathrm{CO}$ animals. The animal group WS125 shown a slight increase in the toe spread in comparison with the CO group and significantly low toe spread in comparison with the LC group of animals. The WD100 group also was not showing significance in the toe spread and can be comparable with the $\mathrm{CO}$ group. This is because this dosage of drug was highly effective in the striatum in protection and so the animals were retaining their gait and co-ordination as like the normal animals.

Distance to Opposite Foot (DOF) (figure-4, chart-2): The distance between the centre of the one forelimb and the centre of the next forelimb print was measured to analyze the gait 
co-ordination of the animals. Less distance to opposite foot was considered as poor gait coordination as per literature. The LC animals had significantly low DOF when compared with the CO group of animals. The SC group of animals had an equal DOF with the $\mathrm{CO}$ animals. The drug group animals WS125 and WD100 were showing slight high DOF. That shows the gait co-ordination was perfect with the WS125 and WD100 animals as their striatum was protected by the drug given.

Table 1: Showing the animal groups used for foot print test.

\begin{tabular}{|c|c|c|c|c|}
\hline S.No & Animal groups & Lesion surgery & $\begin{array}{c}\text { Withania somniferae } \\
\text { thanolic extract } \\
\text { treatment before and } \\
\text { after surgery }\end{array}$ & $\begin{array}{c}\text { Withanolide A } \\
\text { treatment before } \\
\text { and after surgery }\end{array}$ \\
\hline 1 & Control (CO) & NO & NO & N0 \\
\hline 2 & Sham control (SC) & YES (DUMMY) & NO & NO \\
\hline 3 & Lesion control(LC) & YES & NO & NO \\
\hline 4 & WS 125mg (WS 125) & YES & YES & NO \\
\hline 5 & WA100 $\mu$ (WD100) & YES & NO & YES \\
\hline
\end{tabular}

Chart-1: Bar diagram showing the toe spread of the animals in foot print test.

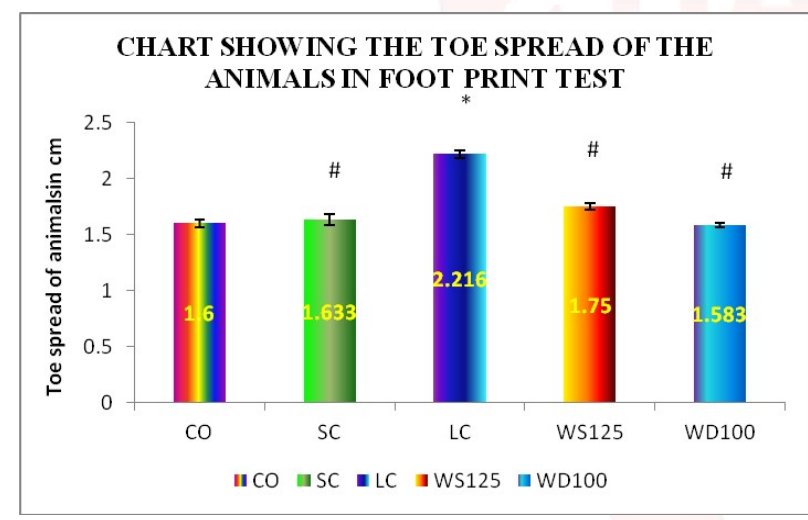

* Indicates significance with CO group \# Indicates significance with LC group

Chart 2: Bar diagram showing the distance to the opposite foot in foot print test.

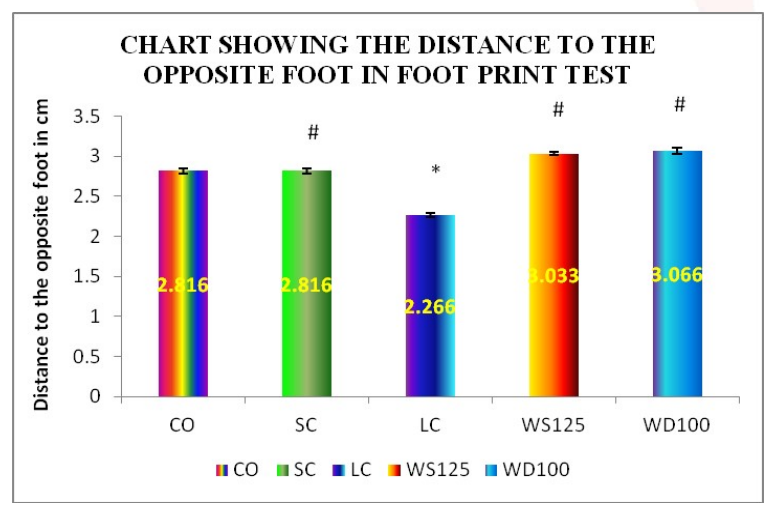

* Indicates significance with CO group \# Indicates significance with LC group
Chart 3: Bar diagram showing the gait difference of the animals in foot print test.

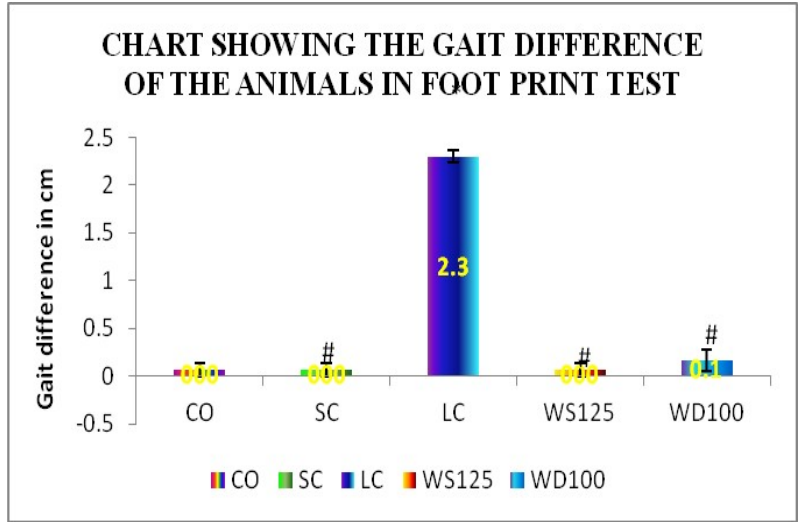

* Indicates significance with CO group \# Indicates significance with LC group

Fig. 1: Foot print test - run way.

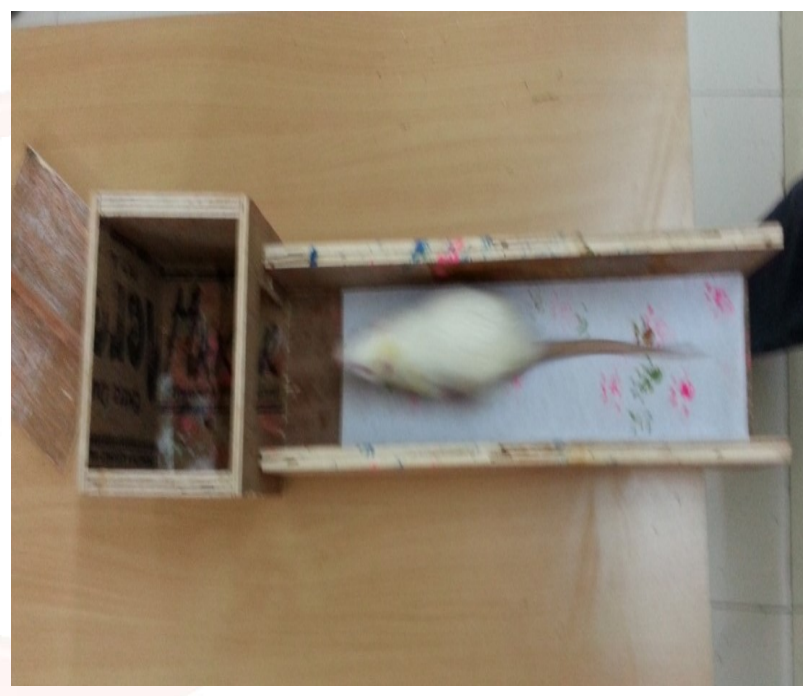

Fig. 2: Figure showing the foot prints of the animals belongs to $\mathrm{CO}$ group.

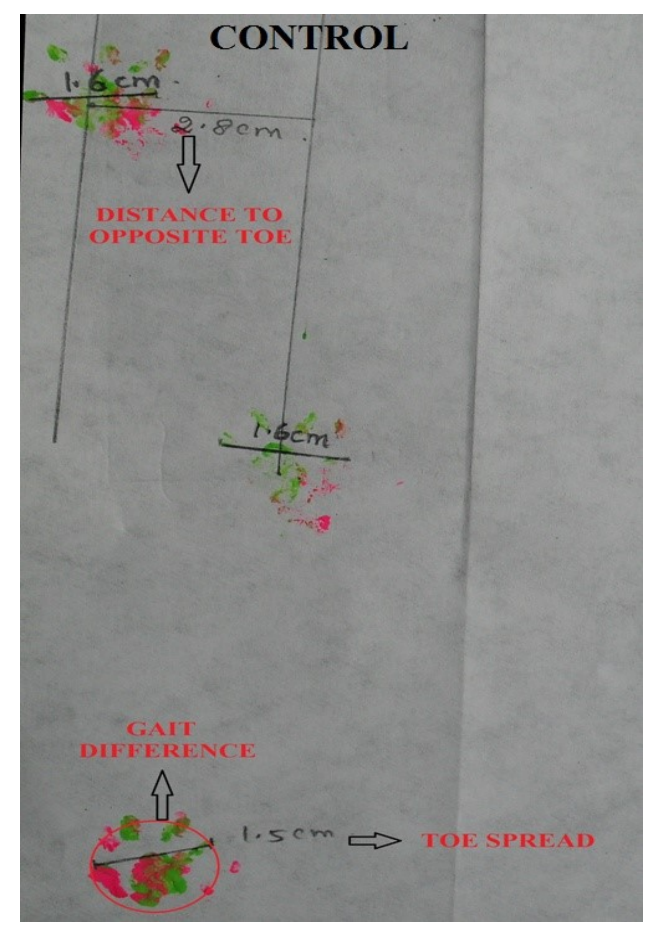


Fig. 3: Showing the toe spread of the animals belongs to different groups.

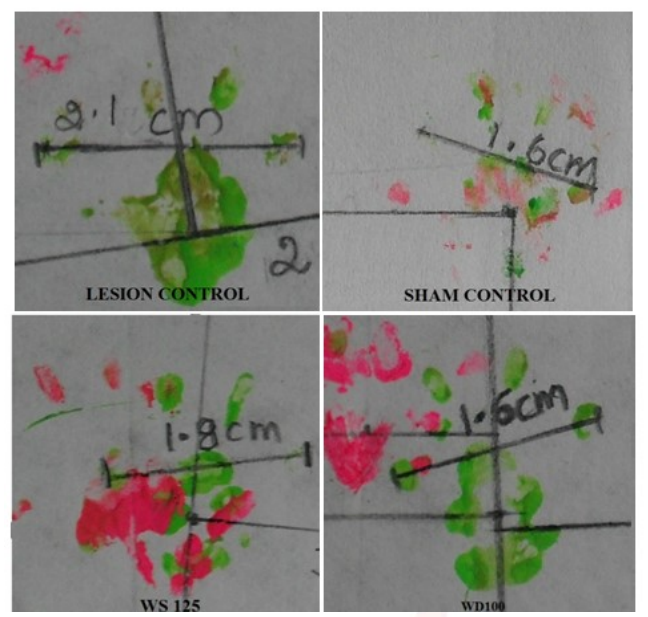

Fig. 4: Showing the distance to the opposite foot of the animals in foot print test.

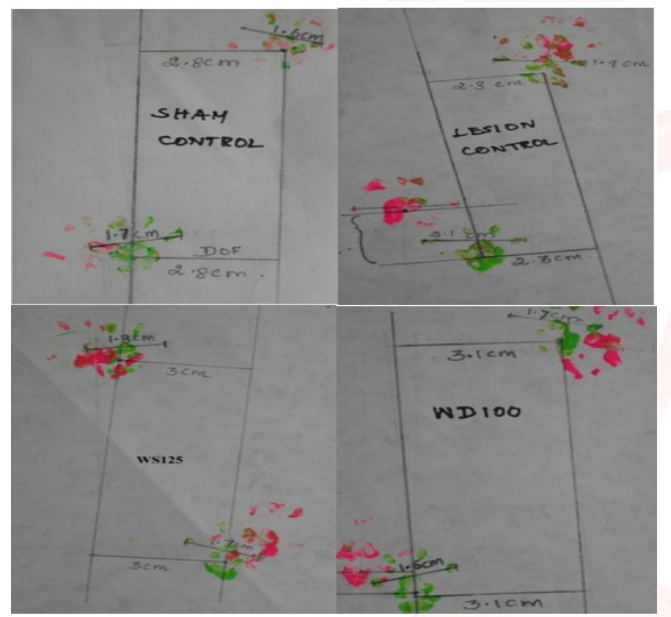

Fig. 5: Showing the gait difference of the animals in foot print test.

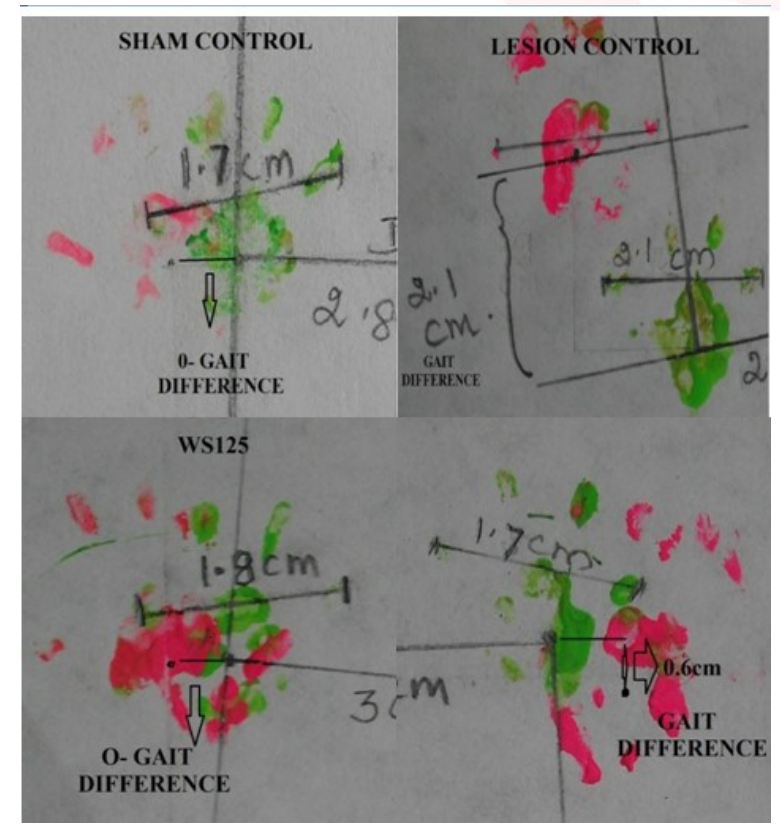

Gait Difference (figure-5, chart-3): Normally the foot print of the hind limbs will overlap the foot prints of the forelimbs. When the footprints did not overlap, the distance between the center of the footprints was recorded as gait difference. High gait difference explains poor balance and gait in-coordination.

The LC animals presented maximum gait difference by showing significant increase in the gait difference in comparison with the CO group of animals. The other animals including the $\mathrm{SC}$ and WS125 were not showing any significant gait difference with the CO animals. The WD100 animals shown a little gait difference which was not so significant with the $\mathrm{CO}$ group. These parameters proved the drugs were acting on the striatum and protecting it from free radicals produced by the lesion.

\section{CONCLUSION}

Vandeputte et al., 2010 [13], investigated the gait parameters of rat models for Parkinson's disease (PD), Huntington's disease (HD) and stroke using the Catwalk method by a novel automated gait analysis test and confirmed that Catwalk analysis proved more sensitive than rotarod test. In this present study the animals belonging to WS125 and WD100 were analyzed for three parameters. Toe spread, distance to the opposite foot and gait difference. The toe spread of the drug group animals were minimum as like the CO animals in that, WD100 showed minimal toe spread. The gait of the animals belongs to WS125 were normal as like the CO group of animals and the WD100 animals were showing a slight difference in their gait but was negligible. In WS125 group the distance to the opposite foot was more than the LC animals and was more equal to the $\mathrm{CO}$ animals but for in WD100 group the foot distance were more than the WS125 group animals. This clearly states both the drugs were effective in neuroprotection and the drug WD100 was bit more effective than the other drug.

As the herbal drugs are free of side effects we can learn the habit of taking these herbal products as food supplements in a very low dose along with our daily drinks or food. This will regularly check our nervous system protect it and will keep it active for a few more years.

\section{ACKNOWLEDGEMENTS}

We are very much thankful to the Dean of Tagore Medical College and the Principal of Tagore 
Dental College- Chennai, for provided us with the necessary facilities to conduct the study. We are also thankful to the technical staffs belongs to the department of Anatomy for their kind help.

\section{Conflicts of Interests: None}

\section{REFERENCES}

[1]. Obeso JA, Rodríguez-Oroz MC, Benitez-Temino B et al. Functional organization of the basal ganglia: therapeutic implications for Parkinson's disease. Mov Disord. 2008;23(3):48-59.

[2]. Stocco, Andrea, Lebiere et al. "Conditional Routing of Information to the Cortex: A Model of the Basal Ganglia's Role in Cognitive Coordination". Psychological Review. 2010:117(2): 541-74.

[3]. Rosenblatt A, Leroi I. Neuropsychiatry of Huntington's disease and other basal ganglia disorders. Psychosomatics. 2000:41(1):24-30.

[4]. Helms A. Shulman L. Chorea, Athetosis, and Ballismus. Demos Medical Publishing. Bookshelf ID: NBK7479.

[5]. Pollard J. A Caregiver's Handbook for AdvancedStageHuntington Disease. Huntington Society of Canada. 2000.

[6]. Jawaid T, Shukla D, Verma J. Anti-inflammatory activity of the plants used in traditional medicines. Int J of Bio Research. 2011;2(4):252 263.

[7]. Elayaraja A, Vijayalakshmi M, Devalarao G. In vitro free radical scavenging activity of various root and rhizome extracts of acorus calamus linn. International journal of pharma and bio sciences. 2010:1(4):301.
[8]. Mohan, Royce. Withanolide compounds as inhibitors of fibrosis and identification of molecular targets for anti-fibrotic drug development. http:// www.freepatentsonline.com/y2008/0032958.html

[9]. Carter RJ, Lione LA, Humby T et al. Characterization of Progressive Motor Deficits in Mice Transgenic for the Human Huntington's Disease Mutation. The Journal of Neuroscience.1999:19(8):3248-3257.

[10]. Venkatramaniah C, Praba AMA, Girija S. A model of temporal lobe epilepsy induced by kainic acid and the behavioral study of the animals treated with Acorus calamus and â-asarone. Journal of Pharmaceutical Sciences and Biomedical Sciences (JPBMS). 2012; 23(27):1-4.

[11]. Longo BM, Mello LE. Supragranular mossy fiber sprouting is not necessary for spontaneous seizures in the intrahippocampal kainate model of epilepsy in the rat. Epilepsy Res. 1998;32(1-2):172-82.

[12]. McGinty JF, Henriksen SJ, Goldstein A, et al. Dynorphin is contained within hippocampal mossy fibers: Immunochemical alterations after kainic acid Administration and colchicines induced Neurotoxicity. Proc. Natl acad. Sci. Usa. 1983;80:589593.

[13]. Vandeputte C, Taymans JM, Casteels C et al. Automated quantitative gait analysis in animal models of movement disorders. BMC Neuroscience. 2010:11:92.

How to cite this article:

A. Mary Antony Praba, C.Venkataramaniah, Kavitha Ganesh, Krishna Eswari. ROLE OF WITHANIA SOMNIFERA AND IT'S ACTIVE PRINCIPLE WITHANOLIDE-A IN BALANCE AND MOTOR CO-ORDINATION BY FOOT PRINT TEST. Int J Anat Res 2018;6(1.1):4788-4793. DOI: 10.16965/ ijar.2017.424 